\title{
Exposure End Date Time
}

National Cancer Institute

\section{Source}

National Cancer Institute. Exposure End Date Time. NCI Thesaurus. Code C83282.

The date and time an exposure has concluded. 